Garay Ibañez de Elejalde, B., Mendiguren Goienola, H., Alvarez Uria, A. \& Vizcarra Morales, M.T. (2017). Los significados otorgados a las relaciones adolescentes desde la comunidad escolar. Revista Electrónica Interuniversitaria de Formación del Profesorado, 20 (3), 179-194. DOI: http://dx.doi.org/10.6018/reifop.20.3.270711

\title{
Los significados otorgados a las relaciones adolescentes desde la comunidad escolar
}

Beatriz Garay Ibañéz de Elejalde, Haritz Mendiguren Goienola, Amaia Alvarez Uria, y Maria Teresa Vizcarra Morales

Universidad del País Vasco/Euskal Herriko Unibertsitatea (UPV/EHU)

\section{Resumen}

Con el uso de las redes sociales han aumentado las situaciones de acoso y violencia de género en la adolescencia. El objetivo de este trabajo es conocer cómo se perciben estas relaciones desde la mirada de la comunidad escolar. Se realizó un estudio de caso para conocer las situaciones vividas en las redes sociales en los centros educativos de VitoriaGasteiz. Se recogieron 470 cuestionarios del profesorado de 30 centros escolares, y se realizaron 7 grupos de discusión, con familias, profesorado y estudiantes. Los resultados muestran que las relaciones entre adolescentes están mediatizadas por los estereotipos; la persistencia del sexismo, el control y la agresividad. Entre las propuestas de futuro el alumnado propone que se hagan foros de debate para compartir aspectos emocionales. El profesorado propone construir un espíritu crítico con el alumnado analizando los mensajes subliminales de las redes. $Y$ las familias proponen revisar actitudes y comportamientos a través de talleres.

Palabras clave

Escuela; redes sociales; relaciones entre pares, género.

\section{The meanings given to adolescents relationships from the school community}

Contacto:

Beatriz Garay Ibañéz de Elejalde, beatriz.garai@ehu.eus, Facultad de Educación y Deporte de la UPV/EHU, Carretera de Lasarte, 71. (01008) Vitoria-Gasteiz. El artículo está vinculado a un proyecto de investigación financiado por el ayuntamiento de Vitoria-Gasteiz y formalizado a través de convenio con la UPV/EHU, con el código y título: CONV14/02, Estudio diagnóstico sobre la situación de la coeducación en los centros educativos de Vitoria-Gasteiz, IP: María Teresa Vizcarra Morales. 


\section{Abstract}

The use of social networks have increased situations of harassment and gender violence in adolescence. The aim of this study was to determine how these relationships are perceived from the perspective of the school community. A case study was conducted to know the situations experienced in social networks in schools of Vitoria-Gasteiz. 470 teacher questionnaires of 30 schools were collected, and 7 focus groups with families, teachers and students were conducted. The results show that relationships between adolescents are mediated by stereotypes; the persistence of sexism, control and aggressiveness. Among the proposals for the future, the students propose discussion forums to share emotional aspects. Teachers propose to build a critical spirit with students by analyzing the subliminal messages of the networks. And families propose to review attitudes and behaviors through workshops.

\section{Key words}

School; social networks; peer relationships; gender.

\section{Introducción y Antecedentes}

En la actualidad han surgido nuevas formas de relación en la adolescencia, que están mediatizadas las modas de las redes sociales. El presente trabajo de investigación tiene por objeto conocer cuáles son los significados y el sentido que las personas adolescentes otorgan a estas relaciones y cuáles son las necesidades que se plantean desde los centros educativos de cara a solicitar nuevas intervenciones en los programas de política social en Vitoria-Gasteiz (España).

\section{Los estereotipos de género en la adolescencia}

Los estereotipos de género son creencias y prejuicios sociales, que uniformizan las características de un grupo social, y que solicitan una manera de ser y de comportarse para los hombres, y otro para las mujeres, llevando a pensar que nadie puede salirse del rol que le ha sido asignado (Arenas, 2006). Las relaciones de la adolescencia en función del género han sido estudiadas en diferentes investigaciones. $Y$ así en la investigación realizada por Ruiz-Pinto con una muestra de 722 estudiantes adolescentes (12-16 años) de Educación Secundaria Obligatoria (ESO) se vio que los estereotipos de género siguen estando presentes en las relaciones adolescentes (Ruiz-Pinto, García y Rebollo, 2013). Mostraron que las chicas eran identificadas con tareas basadas en el cuidado, y los chicos con actividades que implicaban competir, siendo a su vez quienes establecían vínculos más cohesionados y relaciones más fuertes. La asimetría de poder en función del género está presente en la mayor parte de las investigaciones, tal y como se puede ver en el estudio realizado por De la Osa, Andrés y Pascual (2013) sobre la justificación de la violencia en las parejas de adolescentes de $4^{\circ}$ de ESO (15-16 años) donde se mostró que los chicos justifican la aceptación de los estereotipos de género y la violencia ejercida hacia su pareja en mayor medida que las chicas. En otra investigación realizada por Gómez-Valles (2014) se analizó cómo eran entendidos el sexismo, el patriarcado y las alternativas de construcción de la identidad personal desde la perspectiva de la adolescencia. Se constató que tenían un gran desconocimiento en torno a estas temáticas, que reproducían los estereotipos sexistas y que no identificaban las situaciones de violencia de género. Desde ciertos sectores educativos se reivindica la necesidad de fomentar espacios de reflexión sobre la identificación del sexismo benévolo (aquel que es aparentemente inofensivo, pequeños chistes o frases hechas) que está relacionado con la violencia simbólica y el post machismo 
(Arenas, 2013). Todos los estudios revisados recogen que los chicos justifican la división de roles según el sexo, considerando que es algo natural, lo que legitima mantener actitudes sexistas y participar en los casos de acoso, ya que los chicos participan en mayor medida que las chicas. A los chicos se les asocian estereotipos de fuerza, poder, y son benevolentes con el uso de la violencia, mientras que a las chicas les atribuyen debilidad y sumisión (Arenas, 2013; Carretero, 2011; De la Osa et al., 2013; Gómez-Valles, 2015).

Tras los cambios legislativos en materia de género, diferentes investigaciones constatan que las mujeres están asimilando para sí algunos estereotipos masculinos, tales como la agresividad (Quesada y López, 2010), pero, sin embargo, los chicos no están asimilando estereotipos considerados tradicionalmente como femeninos, ante ello se plantea una reconstrucción de los estereotipos en el ámbito escolar.

\section{El amor, la agresividad y la afectividad en las relaciones adolescentes}

Según los estudios revisados, el amor en las relaciones adolescentes suele ir asociado al control, y esto genera actitudes violentas y agresivas dentro de la pareja (Rubio, López, Saúl y Sánchez-Elvira, 2012). Las conductas violentas tienen que ver con la violencia psicológica y verbal, y tienen carácter recíproco. Un porcentaje no desdeñable de chicas y chicos consideraron las agresiones como una conducta "normal" y aceptable en la pareja. Las chicas mostraron mayor tendencia hacia agresiones psicológico-verbales, y expresaron que existe una mayor tolerancia social hacia la pequeña agresión perpetrada por mujeres, mientras que hay gran rechazo social hacia cualquier tipo de agresión física perpetrada por la población masculina. En dicho estudio, la agresión psicológica fue el tipo de violencia más empleada seguida de la violencia física.

Los mitos sobre el amor fueron agrupados por Viejo (2012) en dos dimensiones: la idealización del amor y la vinculación amor-maltrato. En dicho estudio los chicos creen que los celos son un requisito del amor (vinculación amor-maltrato), y las chicas creen más en el amor romántico (idealización el amor), así, se mostró que estos mitos pueden llevar a la aceptación de situaciones límite. En la misma línea, en otro estudio de Rodríguez, Lameiras, Carrera y Vallejo (2013) se demostró que las chicas idealizan más el amor, y los chicos aceptan más el mito de la vinculación amor-maltrato relacionado con actitudes sexistas más hostiles.

\section{Las relaciones adolescentes en las redes sociales}

El uso que se hace de las redes sociales en la adolescencia es un tema preocupante en los foros educativos, se han incorporando al lenguaje escolar términos como bullying, ciberbullying, sexting o grooming. El ciberbullying es el acoso que se ejerce por medio de las redes sociales a través del teléfono móvil o del ordenador, y es consecuencia de la generalización del ciberespacio como medio de socialización (Garaigordobil, 2011). Ese espacio anónimo da sensación de impunidad a quien acosa. Se caracteriza porque se produce en situaciones de asimetría de poder y porque habitualmente se repite (López y Ramírez, 2014).

El número de adolescentes a quienes afecta el cyberbullying va en aumento (Garaigordobil y Oñederra, 2010). En este estudio realizado entre adolescentes vascos recogen que hasta un $69,8 \%$ de adolescentes ha estado implicado en este tipo de situaciones, y lo han sufrido, visto o realizado. Las conductas de acoso más repetidas fueron el robo de contraseñas, las llamadas anónimas con el fin de asustar, difamar y contar mentiras sobre una persona para desprestigiarla, difundir rumores, suplantar el blog de alguien y realizar llamadas ofensivas a través del móvil (Garaigordobil, 2013). 
En otro estudio realizado por el Observatorio Vasco de la Juventud, se recogió que eran las chicas las que se autopercibían como víctimas del sexismo en red, y los chicos se reconocían en más ocasiones como perpetradores de acoso (Estébanez, y Vázquez, 2013). El hecho de realizar más de diez llamadas diarias era considerado por las chicas como una muestra de interés, mientras que para los chicos era una forma de control que ejercían sobre sus parejas. Donde ellas veían amor, ellos veían control. WhatsApp aparece como el medio favorito para comunicarse, pero también el medio favorito para desprestigiar y humillar, y así, las personas participantes en el estudio expresaron que este medio sirve para controlar.

Los trabajos realizado sobre ciberbulling (Acero, 2013; Garaigordobil y Martínez, 2014; Gómez-Viu, 2013; Ortega, Del Rey y Casas, 2013; Valverde, 2013) proponen estrategias para minimizar sus efectos.

\section{La actitud del profesorado ante el ciber-acoso}

El profesorado suele desconocer los casos de acoso del aula (Serrano y Pérez, 2011), y cuando son consientes de lo que ocurre no saben cómo actuar. Esto suele llevar a negar el problema, a hacer como que no lo ven, o buscar estrategias inconscientes de evitación. Las investigaciones previas muestran que el profesorado carece de conocimientos y de formación específica para tratar las situaciones de acoso (Castro, Sánchez, y Sánchez, 2014). Y sin embargo, la tutoría es vista como un espacio de trabajo para la mediación (RodríguezGómez, 2008). Otras iniciativas proponen implementar la reflexión y debate en los espacios escolares a través de grupos reflexivos para docentes (Domínguez, López, Pino y Álvarez, 2013). En este sentido, distintas voces señalan que cada vez es más necesario que las políticas educativas y las acciones que se realizan en el ámbito educativo sean "espacios de reflexión donde los docentes compartan experiencias y saberes con sus colegas" (Cuevas, 2015, p. 69). Por ello, se reclama la necesidad de revisar cuál es la actitud del profesorado ante las situaciones de acoso.

\section{Objetivos}

El presente estudio realizado con el fin de incidir en las políticas sociales del ayuntamiento de Vitoria-Gasteiz, pretende:

- Comprender cómo perciben las y los estudiantes, familiares y profesorado las relaciones entre adolescentes.

- Reconocer qué estereotipos de género existen, para comprender cómo entienden la agresividad, la afectividad, el amor y el control.

- Indagar sobre cómo perciben todas estas situaciones en las redes sociales, así como, sí en las relaciones entre chicas y chicos se dan asimetrías de poder.

Detectar qué actitudes toma el profesorado ante las situaciones de acoso de cara a programar acciones formativas.

\section{Método}

\section{Participantes}

Participan en la investigación 30 centros escolares públicos y concertados (0-18 años) de Vitoria-Gasteiz. Participan 470 docentes, 26 estudiantes, 6 padres y 16 madres. 


\section{Instrumentos}

Se utilizó un cuestionario de respuesta abierta que fue enviado al profesorado y a las direcciones de los centros. Se recogieron 421 cuestionarios del profesorado y 49 de equipos de dirección. Con el cuestionario se quería hacer una aproximación a la percepción que el profesorado tenia sobre las relaciones que su alumnado mantenía en las redes sociales para observar si se daban comportamientos agresivos, actitudes estereotipadas, relación amor-control, situaciones de acoso y sus formas. Una vez realizados y analizados los cuestionarios, se realizaron los guiones de los grupos de discusión tomando como ejes estas mismas cuestiones, así como qué actuaciones se proponían ante este tipo de casos. Se llevaron a cabo 7 grupos de discusión ( 2 con profesorado, 2 con familias, y 3 con escolares, 60 personas).

\section{Diseño}

La presente investigación se llevo a cabo en el curso 2014/2015. Se trata de una investigación con enfoque cualitativo e interpretativo, ya que se centra en interacciones con las personas participantes en la investigación, para aproximarse a la realidad (Monarca, 2015). El cuestionario fue utilizado para descubrir qué significados otorgaban las personas participantes a los diferentes constructos (Goetz y Lecompte, 1988), y para rescatar apreciaciones más sutiles (Bisquerra, 2004). Del análisis de los cuestionarios recogidos surgió el guión de los grupos de discusión, que ha supuesto una estrategia metodológica básica para recoger información sobre las temáticas que no quedaron claras en el transcurso de la investigación, ya que permitió formular preguntas desde un planteamiento comunicativo y dialógico con los y las participantes (Aubert, García y Racionero, 2009).

\section{Procedimiento de análisis}

Se realizó un análisis de contenido, fundamentado en las significaciones semánticas. Se utilizó como herramienta analítica un sistema categorial con nodos axiales que fue construido siguiendo criterios inductivo-deductivos, esto es, se apoyó en la revisión teórica y en aquellos aspectos emergentes que surgieron de las aportaciones de cada participante. Para analizar la información se utilizó el soporte digital NVivo11. Las categorías resultantes son (ver tabla 1 en los resultados), relaciones en la adolescencia, relaciones en las redes sociales, comportamiento del profesorado ante situaciones de acoso sexista.

La codificación de la información: si proviene de un cuestionario, presenta un código de 4 números, donde las dos primeras cifras se corresponden con el centro escolar y las dos siguientes con la persona informante; a continuación aparece las siglas de la etapa (Educación Infantil, El, Educación Primaria, EP, Educación secundaria, ESO...); y por último, V ó M (varón o mujer). Si se trata de información vertida en los grupos de discusión el código es GD, a continuación aparece la audiencia que ha hecho tal afirmación (Alumnado, A, Profesorado, P, Familias, F) y un número que corresponde con el orden en que se celebran los grupos de discusión de cada audiencia.

\section{Resultados}


En la investigación desarrollada vemos qué sucede con los estereotipos en el uso del lenguaje, las imágenes, los insultos, y los discursos homófobos o racistas, y cómo esto se refleja en las redes sociales.

Tabla 1.

Herramienta de análisis: Sistema categorial utilizado

\begin{tabular}{|c|c|c|}
\hline Dimensiones & Categorías & $\begin{array}{l}N^{\circ} \text { de intervenciones } \\
\text { recogidas en los GD }\end{array}$ \\
\hline \multirow{4}{*}{$\begin{array}{l}\text { Relaciones en la adolescencia en } \\
\text { general }\end{array}$} & Estereotipos & 21 \\
\hline & Asimetrías de género & 19 \\
\hline & Agresividad y afectividad & 23 \\
\hline & Amor relacionado con control & 12 \\
\hline \multirow[t]{2}{*}{$\begin{array}{l}\text { Relaciones de la adolescencia en } \\
\text { las redes sociales }\end{array}$} & $\begin{array}{l}\text { Potencial de difusión de } \\
\text { situaciones de acoso en las } \\
\text { redes y sexismo. }\end{array}$ & 29 \\
\hline & Características del acoso & 17 \\
\hline $\begin{array}{l}\text { Actuaciones del profesorado } \\
\text { ante las situaciones de acoso }\end{array}$ & Actuaciones & 421 \\
\hline
\end{tabular}

\section{Las relaciones de la adolescencia}

Se presentarán cómo son percibidos los estereotipos en la adolescencia, cómo son las asimetrías de poder y cómo son entendidos agresividad, afectividad y control.

Cómo son percibidos los estereotipos de género en la adolescencia:

Las personas participantes en este estudio muestran que los estereotipos de género no se han erradicado en la escuela. El profesorado expresa que al inicio de la educación primaria (6 años) ya se han interiorizado los roles de género. El lenguaje que utilizan es sexista, y aunque la mayor parte de las veces no son conscientes del alcance de las palabras que utilizan, saben que molestan. Los estereotipos relativos a las capacidades y habilidades físicas son los más arraigados. Asignan género a los trabajos, los colores y los gustos.

Puede que haya actitudes sexistas que provienen de lo que aprenden, por ejemplo, cuando se necesita la fuerza son los chicos los que tienen que actuar porque las chicas no tienen fuerza. (0406_EP_M).

Los estereotipos se reafirman a medida que se sube de etapa. Se utilizan calificaciones sexistas, que suelen ser positivas en masculino y negativas en femenino, en especial en secundaria (12-16 años), los insultos están incorporados al lenguaje diario de la adolescencia. Aparecen palabras homófobas y chistes. Expresan, además, que todo está contaminado, carteles, anuncios.... 
Los chicos hablan de las chicas llamándolas "Jaca, jamba, jamona, conejito...", estas palabras forman parte de su manera natural de comunicarse (2610_ESO_V).

Para el profesorado estas frases provienen de los mensajes escuchados en televisión, los interiorizan con facilidad, creen que en las escuelas no se ha realizado ningún estudio sobre lo que pasa con el uso del lenguaje. El alumnado expresa que los comentarios suelen partir de los chicos para conseguir la reacción de las chicas.

Hay veces que los chicos dicen algo en clase, para ver si alguien se ríe, o si alguien mira... para hacer una gracia. (GD_A2)

El profesorado cree que la adolescencia no es nada crítica con la situación, que su discurso está mediatizado, y que repiten lo que ven.

Intento hablar con el alumnado, y a veces, parece que todavía están en la prehistoria, aunque hayan visto que sus madres trabajan, son como trogloditas (GD_P1).

Las familias se centran en que la manera de hablar no siempre reproduce esa discriminación discursiva, y encuentran resistencias hacia la paridad.

Yo no me considero machista y creo que el lenguaje no es neutro, ¿eh? ¡Ojo!,... pero me cuesta. (GD-F2).

\section{Asimetría de género}

Las relaciones de poder son asimétricas en función del género, y eso provoca conflictos, ante los que el profesorado no sabe cómo actuar, y reclama formación para afrontarlo.

Ante las situaciones de conflicto no se toman medidas ante diferentes comportamientos. Nos falta formación ya que no sabemos qué hacer ante determinadas situaciones. (GD_P1_M).

El alumnado encuestado expresa que ser chico o chica tiene sus ventajas y desventajas, y que a la mujer se le otorga un papel de debilidad.

A las chicas los padres no nos dejan salir hasta tarde, porque igual nos violan, pero con los chicos piensan que son más fuertes y que se defenderán. Nos enseñan desde pequeñas que somos ‘Barbies' y que los chicos son más fuertes. Nos lo dicen desde pequeñas y nos lo acabamos creyendo. (GD_A2M).

En las clases se dan situaciones desagradables relacionadas con comentarios que realizan los chicos sobre las chicas, y aunque, a veces, la intención no es hacer de menos a las mujeres, lo hacen. Las percepciones de las chicas y de los chicos son diferentes.

A mí que me dijeran que no era tan femenina por no llevar faldas, me dolió mucho, y el resto de la clase no se dio cuenta, y que al replicar me dijeran que no era para tanto, todavía me hizo sentirme peor. (GD_A1M)

Son esos chistes que haces para hacer la gracia, no para hacer daño a nadie y que en realidad son machistas, y que están descalificando a la mujer aunque no lo hace con esa intención y no piensan en lo que está diciendo. (GD_A3V).

A las chicas les genera miedo e inseguridad hacer frente a las actitudes sexistas del profesorado.

Si no dices nada, al final todo sigue igual, pero decirle algo a algún profesor es mucho más difícil. (GD_A2M) 
Para las familias el reparto de roles se aprende socialmente, por ello, aunque se les dé una formación coeducativa, los chicos siguen jugando al fútbol aunque no les guste, y que si una chica juega al fútbol, como rompe las normas generales será objeto de bullying, y recibirá el castigo del estigma social.

Yo sigo viendo a mi hijo cómo se comporta en el patio, no le gusta el fútbol y tiene que jugar porque si no se encuentra aislado en el grupo de clase. I... está muy castigado que un niño juegue con niñas. (GD_F2)

Se van perdiendo los valores femeninos y reforzando lo masculino, lo femenino va dejando paso al rol hegemónico masculino, porque va ligado al poder.

Porque lo femenino a lo mejor siempre ha estado un poco devaluado. La propia chica entre lo masculino y lo femenino, si lo masculino implica poder, liderazgo, en vez de recuperar lo femenino y reivindicarlo, lo está desterrando. (GD_P2)

Estas relaciones asimétricas de poder se ven reflejadas en la agresividad de las relaciones adolescentes.

\section{Agresividad y afectividad}

Relacionado con lo anterior, el profesorado expresa que las chicas están adoptando modelos masculinos de agresividad, y cada vez es más habitual ver a chicas pelearse a puñetazos. Expresan que la agresividad física ya no es solo patrimonio de los chicos, y la afectividad se está debilitando.

Esas peleas que antes solo se veía en chicos y que en las chicas era impensable, las chicas era gritarse o tirarse de los pelos un poco. Pero yo creo que sí que se están dando niveles altos de agresividad. (GD-P2).

Las familias afirman que en las relaciones entre chicos y chicas, la afectividad y la agresividad son diferentes atendiendo a estereotipos de género. Son más habituales los abrazos entre chicas, y una relación más agresiva entre chicos.

En el patio se ve claramente, yo recuerdo a niñas de la clase de mi hija mayor, que se encontraban en el patio y se daban un abrazo, y ves a los chicos y se dan un par de tortas..., es un poco esa forma de relacionarse que a los chicos pocas veces les ves abrazarse. (GD_F1M)

El alumnado piensa que los chicos no hablan de sus sentimientos porque tienen dificultades para gestionarlos. Proponen desarrollar talleres sobre emociones para reflexionar en clase y mejorar las relaciones y la empatía. Creen que la expresión de los sentimientos evitará tantos episodios de violencia.

Con los talleres de emociones se puede entender a los demás, ponernos en su lugar, y eso es muy bueno para evitar el sexismo y el machismo. (GD_A2M).

Los hombres que pegan no saben expresar sus sentimientos, si les ayudan a expresar cosas como que están enfadados, a lo mejor habría menos violencia. (GD_A2M).

Creen que la escuela debería ser un lugar donde poder expresar los sentimientos. El alumnado comenta que en Educación Secundaria, se viven situaciones de agresividad, pero que no suelen ser mixtas, son entre personas del mismo sexo.

En primero había muchas peleas, de chicas con chicas y de chicos con chicos, no eran mixtas. Muchas veces tenía que ver con que un chico le ha dicho algo a su novia. (GD_A2). 
El alumnado reivindica las tutorías como un espacio apropiado para hablar de sus sentimientos, y prevenir estas situaciones.

Es bueno poder hablar en tutoría de lo que nos pasa, de lo que sentimos, y no tanto de otros temas que no nos importan tanto, bueno ahora en bachillerato ya no tenemos tutorías. (GD_A2).

\section{Amor y control}

Se definen las relaciones amorosas entre adolescentes como muy controladoras, aunque ellas y ellos lo tomen como algo normal, y no le den importancia. En los GD se reforzó la idea del amor romántico. A veces confunden el amor con el control, 'está tan pendiente de mí, que es porque me quiere mucho'. El profesorado en los GD cree que puede ser debido a las series televisivas y los modelos de las redes sociales.

Para cuando nos damos cuenta ya es tarde, en cuarto de la ESO es demasiado tarde para trabajar esto. (GD_P1)

Hablan mucho por Facebook utilizando todos los estereotipos de las series de moda que han sido calificadas como 'cutres' por las familias.

Uno de los temas que nos preocupa son esas primeras relaciones amorosas en donde a veces hay riesgos serios de desigualdad, pues puedan llevar a unas primeras elecciones erróneas que marcarán futuras elecciones, y que, a lo mejor, con el tiempo pueden terminar en agresiones. (GD_P2).

Las relaciones entre jóvenes, tal y como nos comenta el alumnado en los GD, están habitualmente asentadas en relaciones de poder.

En el instituto hay una pareja, el chico tiene controlada a su chica, le mira las conversaciones del móvil, los WhatsApp, con quién queda... no le deja quedar con nadie, no le deja ser ella misma, para mí eso son malos tratos. (GD_A2).

\section{Relaciones de la adolescencia en las redes sociales}

Cuando las relaciones adolescentes negativas llegan a las redes sociales, aumenta su potencial de difusión, de utilizar expresiones sexistas y acosar sin dar la cara.

\section{Potencial de difusión y sexismo}

En las pantallas también se genera el mismo sistema de exclusión y dominación sexo/género que existe en la sociedad. Sobre los mensajes que aparecen en las redes sociales a las familias les preocupa no poder controlar lo que ocurre, ya que la mayor parte de los modelos que se dan son muy sexistas y violentos, no saben cómo actuar.

Con el móvil acceden a un mundo difícil de controlar, es muy complicado pero deberíamos trabajar con esto. Internet manda unos mensajes terribles, habría que explicar a la juventud que la mujer no tiene porqué aparecer de escaparate al lado de un coche caro. (GD_F2M).

Gracias a las redes sociales un pequeño problema social puede alcanzar una dimensión enorme en poco tiempo. El profesorado expresa que antes los insultos eran verbales y llegaban a un grupo reducido de gente, ahora con el WhatsApp la gente tiene redes y se 
difunde cualquier insulto con rapidez, además pueden participar personas ajenas que hacen más grande la bola de nieve.

El mismo problema ocurre también en sus relaciones de pareja, cuando la relación es compleja y controladora, son fáciles de identificar, pero no de solucionar. (GD_P1)

Presentan mucho miedo a cómo se están utilizando las redes sociales, ya que el alumnado no tiene formación para saber dónde se meten, y el profesorado tampoco. En los centros educativos no hay ningún protocolo sobre qué hacer con los delitos que se están empezando a dar en WhatsApp. Las direcciones de los centros expresan que deberían tener protocolos para hacerle frente, pero la escuela no debería ser la única responsable.

En estos mensajes no siempre es fácil identificar los mensajes ocultos. Lo femenino está en horas bajas, los discursos que sobrevaloran lo masculino son cada vez más fuertes, la masculinidad tiene mucha fuerza en las redes, ya que la imagen de la mujer se utiliza constantemente como objeto. Se hace poco por cambiar esto, y así nos lo comenta el profesorado.

Es que incluso en esa franja de dibujos animados, los niños y las niñas de 8 años ven cosas que son... no sé si la palabra es denunciable, y pienso: ‘'Nadie ve esto antes de proyectarlo?' (GD_P2).

Los medios de comunicación no ayudan en las situaciones de violencia de género, y para el alumnado las mujeres se llevan la peor parte, denuncian la poca implicación de las instituciones.

\section{Acoso}

Lo mismo ocurre cuando suben fotos a la red, en los talleres de formación para la prevención de peligros en las redes sociales se trabajan estas situaciones, porque pueden darse situaciones de violencia, que el alumnado no identifica como tales hasta que es demasiado tarde. Las familias y el profesorado echan en falta recursos para ayudar al alumnado con protocolos que detecten estas situaciones.

Los familiares dicen: 'Igual no denuncio porque es peor para la chavala o el chaval...' Pero es un delito, si te hubiesen dado una paliza seguramente hubieses denunciado. Bueno, es complicado y yo creo que es una sugerencia a instituciones, un protocolo me parecería adecuado (GD_P2).

El profesorado no sabe en qué casos tiene obligación de denunciar, desconocen qué consecuencias puede tener grabar al alumnado, o subir lo grabado a la red.

Pero es que empezamos a rozar lo que son delitos realmente, y lo recogido en el código penal. Y los centros educativos no tienen por parte de Delegación de Educación ninguna indicación, no te dicen 'Éste es el protocolo que tenéis que seguir'. E (GD_P2)

Las redes sociales influyen mucho en las relaciones de la adolescencia, una utilización no adecuada puede suponer un comportamiento inapropiado e incluso, violento. El alumnado expresa que hay quienes tienen cuentas ocultas, a través de las que es fácil realizar amenazas, organizar peleas... hay gente que se envalentona cuando no tiene que dar la cara.

Es más fácil que haya un grupo que vaya todo el rato contra una persona porque están detrás de una pantalla, y pueden tener un perfil falso perfectamente. Hay gente a la que igual no le afecta, pero luego hay gente que lo pasa verdaderamente mal. . (GD_A3). 
Muchas de estas situaciones no se denuncian, en la mayor parte de las ocasiones, por miedo.

Hay veces que ves que alguien lo está pasando mal, y tienes ganas de ayudarle, pero piensas que se van a poner el resto en tu contra y al final te quedas al margen. (GD_A2)

\section{Actuaciones del profesorado ante las situaciones de acoso}

Cuando a los equipos directivos de los centros se les pregunta si han recibido denuncias de acosos sexistas, solo un $11,8 \%$ de las direcciones afirma haber tenido alguna denuncia, el $51 \%$ afirma no haber tenido ninguna, y $37,2 \%$ no sabe o no contesta (ver figura 1). En estos casos, la respuesta habitual de los centros es acudir a la familia, a los servicios de apoyo al profesorado, a los servicios sociales o a la policía.

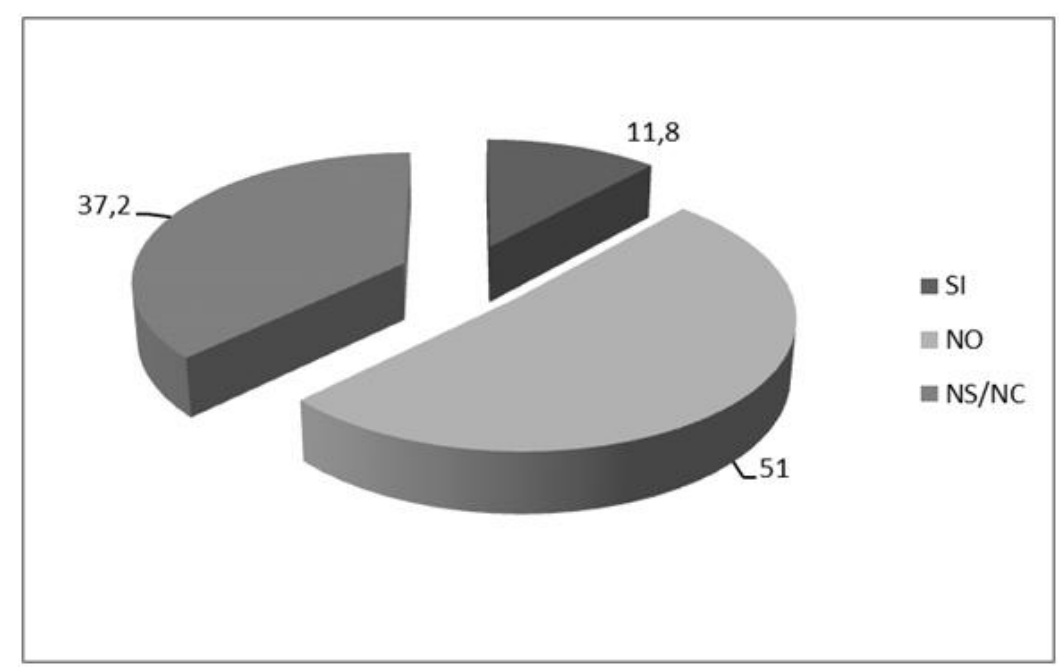

Figura 1. Denuncias de acoso sexista recibidas por los equipos directivos de los centros.

Cuando se le pregunta al profesorado qué se suele hacer en los casos de acoso por razones de género, las respuestas son muy diversas. Para analizar la información que proporcionan hemos realizado un mapa (figura 2) que recoge el posicionamiento de los docentes en base a dos ejes, la implicación o no implicación de los mismos en la intervención ante el acoso, y en el otro eje sitúa de dónde parte la ayuda, si la ayuda se centra en el docente, o si esta se externaliza, se deja en manos de otros profesionales. 


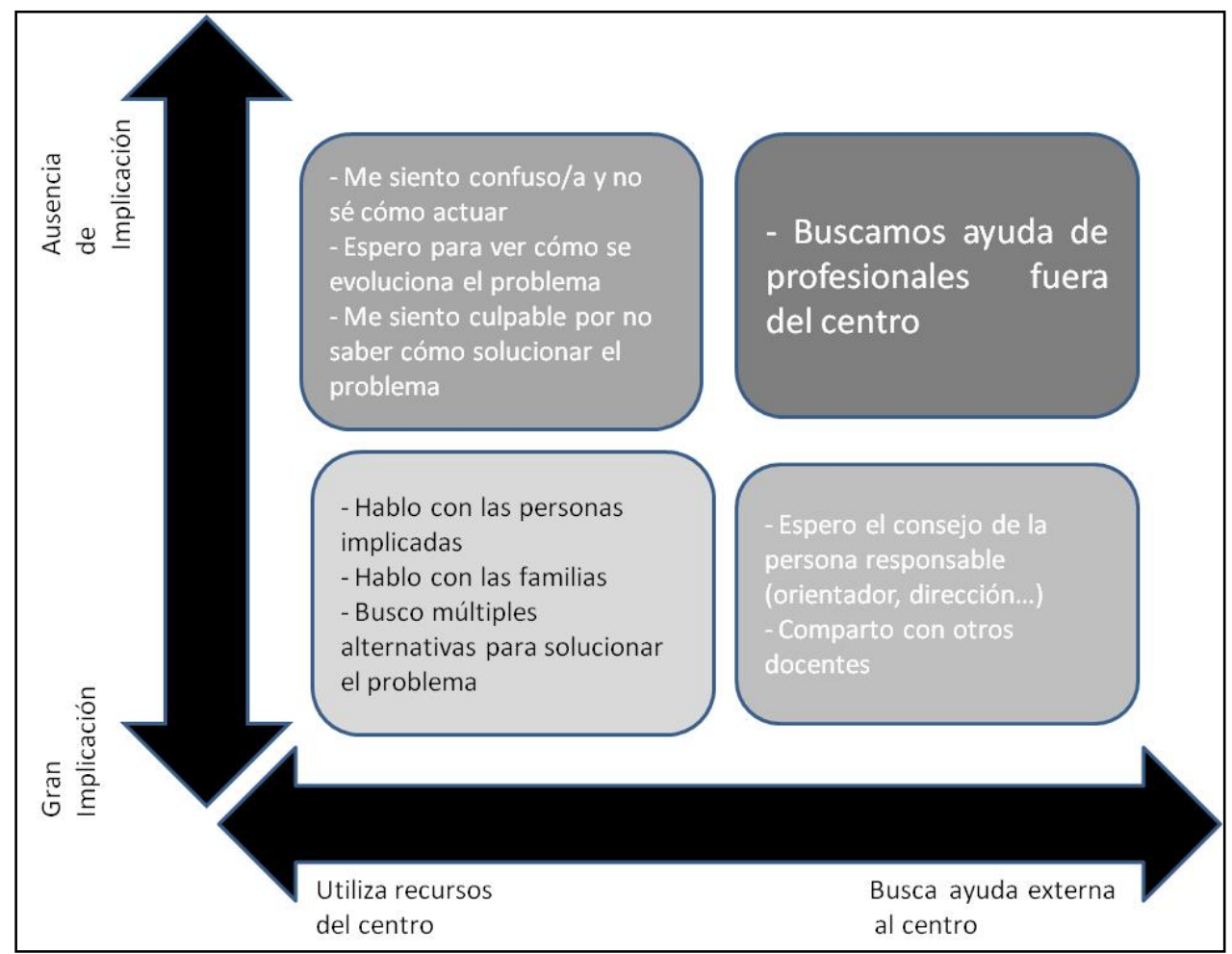

Figura 2. Mapa de posiciones del profesorado ante el acoso

En la parte de abajo del extremo izquierdo encontramos docentes que se implican y que lideran la búsqueda de soluciones, y en la parte de arriba docentes que no se implican en la gestión de las ayudas. En el otro eje encontramos docentes que se implican en la búsqueda de soluciones pero no lideran esta ayuda, sino que la dejan en manos de otros profesionales. $Y$ finalmente en el extremo superior derecho están los docentes que ni se implican, ni lideran la ayuda, sino que externalizan la búsqueda de soluciones en profesionales ajenos al centro escolar.

\section{Discusión y conclusiones}

Según los resultados de este estudio las relaciones sociales de la adolescencia avanzan muy lentamente en la superación de los estereotipos de género, y existe gran control en las relaciones amorosas. El profesorado ha detectado una interiorización muy temprana de los estereotipos, lo que hace muy difícil trabajarlos en secundaria cuando están ya afianzados, al igual que recogen varios autores (Arenas, 2013; Carretero, 2011; De la Osa et al., 2013). En este estudio se recoge que una parte del profesorado no se cuestiona si la información que se maneja en la escuela está estereotipada o no, tal y como ocurría en otros estudios (Arenas, 2013; Ruiz-Pinto et al., 2013). Entre la juventud dicen que existe un "sexismo benevolente" o paternalista como una tendencia arraigada entre los chicos. El profesorado muestra descontento ante la tarea de luchar contra los mensajes que transmiten los medios de comunicación, esto mismo ocurría en los estudios de Garaigordobil desde 2010 a 2014. Los modelos que transmiten las redes son consumidos y asumidos rápidamente por una juventud necesitada de modelos para recibir la aceptación de sus iguales. 
El profesorado detecta que las asimetrías de género no se han superado y esto lleva a una pérdida la valoración positiva de lo femenino, por ello perciben un aumento de la agresividad y menor expresión de la afectividad. La juventud de este estudio reconoce que tienen cierta identificación con el amor romántico donde se confunde control con amor, y que las chicas idealizan el amor, y los chicos vinculan amor a control y maltrato (Rubio et al., 2012; Viejo, 2012).

Familias y profesorado detectan sesgos sexistas en las formas de relación, en la agresividad y en las emociones de la juventud. Entre los chicos sigue persistiendo el abuso físico mientras que en las chicas predomina el abuso social. Expresan que se acepta mejor la expresión y las muestras de agresividad en los chicos que en las chicas, aunque cada vez se detectan más casos de chicas que utilizan la violencia física para resolver los conflictos. Existe una asimilación por parte de la mujer de los estereotipos masculinos, y se considera que debe haber un trabajo en valores, en especial es el alumnado quien solicita que se desarrollen programas educativos (Ortega et al., 2013; Valverde, 2013). El profesorado afirma que el alumnado consume sexismo homologado y empaquetado a través de la programación televisiva y los videojuegos, y les gustaría que la administración regulara los programas y la publicidad (Castro et al., 2014).

El profesorado expresa encontrarse fuera de juego ante el aumento de problemas relacionales de acoso, y ciberbullying, expresan tener dificultades para detectarlos y echan en falta la implicación de las instituciones educativas para generar protocolos de actuación. Se quejan de ser los últimos en enterarse de estas situaciones. Ven necesaria una mayor colaboración y comunicación entre familias, profesorado, alumnado e instituciones educativas, coincidiendo en esto con estudios anteriores. (Castro et al., 2014; Serrano y Pérez, 2011).

La mayoría de los y las docentes del estudio muestran implicación para liderar la búsqueda de soluciones, aunque hay quien externaliza la búsqueda de soluciones con profesionales fuera del entorno escolar ante su falta de capacidad. Reclaman una mayor formación práctica en estos temas, y que ésta se centre en casos diarios, para que las situaciones leves no sean pasadas por alto, y no desemboquen en situaciones graves. El alumnado del presente estudio tiene la misma percepción, coincidiendo con otros estudios (Castro et al., 2014).

La tutoría es vista como un espacio de trabajo para la mediación, algo similar a lo que ocurre en otros estudios (Rodríguez-Gómez, 2008). Alumnado y profesorado reclaman apoyo institucional para desarrollar entornos escolares seguros e incentivar la formación del profesorado dotándolo de recursos para trabajar en este ámbito y disponer de espacios donde realizar consultas, denuncias o confidencias (Ortega et al., 2013; Serrano, y Pérez, 2011).

Las tres audiencias de este estudio ponen en valor la capacidad reflexiva y crítica de la frente a estos modelos, expresando que para ello, hay que dedicarle tiempo y los recursos necesarios, y no tratarlo como un tema puntual en una tutoría. Solicitan que sea un tema transversal en todo el proyecto educativo del centro, tal y como apuntan otros estudios (Rodríguez et al., 2013).

Prospectiva de la investigación: A instancias de las diferentes audiencias consultadas de cara a futuros estudios el alumnado propone realizar foros de debate en los centros donde 
se traten temáticas relacionadas con la educación socioemocional y los valores para compartir y aprender. El profesorado propone analizar mediante las tutorías los anuncios televisivos, y los mensajes que llegan de las redes sociales de manera subliminal para intentar construir un espíritu crítico con las chicas y los chicos, que permita prevenir ciertas actitudes y comportamientos. Las familias así mismo proponen que se realicen talleres en horario extraescolar con el mismo fin. En este momento parece que lo que ocurre con las redes sociales es incontrolable, por ello es una tarea que queda por abordar.

\section{Referencias}

Acero, S. (2013). Cibermanagers: Una experiencia de aprendizaje y servicio para la inserción segura y saludable de las TICS en el aula y en el entorno escolar en general. Revista digital de la Asociación Convives, vol.3, 48- 52. Recuperado el 22 de mayo de 2015 desde http://www.educatolerancia.com/pdf/ACOSO\%20ENTRE\%20IGUALES_CIBERACOSO_ \%20Revista\%20Convives.pdf

Arenas, M.G. (2006). Triunfantes perdedoras: La vida de las niñas en la escuela. Barcelona: Graó.

Arenas, L. (2013). Sexismo en adolescentes y su implicación en la violencia de género. Boletín Criminológico. Instituto andaluz interuniversitario de Criminología, vol.144, 1-5.

Aubert, A., García, C. y Racionero, S. (2009). El aprendizaje dialógico. Cultura y Educación, vol.21 (2), 129-139.

Bisquerra, R. (2004). Metodología de la investigación educativa. Madrid: La Muralla.

Carretero, R. (2011). Sexo, sexismo y acoso escolar entre iguales. Revista Complutense de Educación, vol.22 (1), 27-43.

Castro, S., Sánchez, M.I. y Sánchez, J.H. (2014). El rol del profesorado en el manejo de la violencia escolar. Revista Via Inveniendi et Judicandi, vol.9 (1), 51-73.

Cuevas, Y. (2015). Representaciones sociales de la reforma educativa básica. La visión de los directivos. Perfiles educativos, vol.38 (147), 67-85.

De la Osa, Z., Andrés, S. y Pascual, I. (2013). Creencias adolescentes sobre la violencia de género. Sexismo en las relaciones entre adolescentes. European Journal of Investigation in Health, Psychology and Education, vol.3, 265-275. doi: 10.1989/ejihpe.v3i3.49

Domínguez, J., López, A., Pino, M. y Álvarez, E. (2013). Violencia escolar: la díada interpersonal profesorado-alumnado. European Journal of Investigation in Health, Psychology and Education, vol.3 (2), 75-86.

Estébanez, I. y Vázquez, N. (2013). La desigualdad de género y el sexismo en las redes sociales: una aproximación cualitativa al uso que hacen de las redes sociales las y los jóvenes de la CAPV. Vitoria-Gasteiz: Colección Gazteak Bilduma del Gobierno Vasco. 
Garaigordobil, M. y Oñederra, J.A. (2010). Los centros educativos ante el acoso escolar: actuaciones del profesorado, acciones sancionadoras y actividades de prevención. Información Psicológica, vol.99, 4-18.

Garaigordobil, M. (2011). Prevalencia y consecuencias del cyberbullying. International Journal of Phychology and Psychologial therapy, vol.11 (2), 233-254.

Garaigordobil, M. (2013). Cyberbullying: Prevalencia en el País Vasco, conexión con variables personales y familiares, y programa de prevención e intervención. Revista digital de la Asociación Convives, vol.3, 45-46. Recuperado el 22 de mayo de 2015 desde http://www.educatolerancia.com/pdf/ACOSO\%20ENTRE\%20IGUALES_CIBERACOSO $\% 20$ Revista\%20Convives.pdf

Garaigordobil, M. y Martínez, V. (2014). Efecto del Cyberprogram 2.0 sobre la reducción de la victimización y la mejora de la competencia social en la adolescencia. Revista de Psicodidáctica, vol.19 (2), 289-305.

García-Meseguer, M.A. (2008). El sexismo en el uso del lenguaje reflejado en la escritura. Un estudio en el aula. Murcia: Universidad de Murcia.

Goetz, J.P. y Lecompte, M. (1988). Etnografía y diseño cualitativo en investigación educativa. Madrid: Morata.

Gómez-Viu, C. (2013). Actividades de prevención sobre el Cyberbullying. Revista digital de la Asociación Convives, vol.3, 53-63. Recuperado el 22 de mayo de 2015 desde http://www.educatolerancia.com/pdf/ACOSO\%20ENTRE\%2OIGUALES_CIBERACOSO_ \%20Revista\%20Convives.pdf

Gómez-Valles, E. (2015). Perspectiva social de la infancia en algunas comunidades del país vasco (opiniones de las y los adolescentes de Santurtzi sobre el sexismo). Bilbao: UPV-EHU (Trabajo de Fin de Grado).

López, L. y Ramírez, A. (2014). Medidas disciplinarias en los centros educativos: ¿suficientes contra el acoso escolar? Perfiles educativos, vol.26 (145), 32-50.

Monarca, H. (2015). Políticas, prácticas y trayectorias escolares. Dilemas y tensiones en los procesos de inclusión. Perfiles educativos, vol.37 (147), 14-27.

Ortega, R., del Rey, R. y Casas, J.A. (2013). Redes Sociales y Cyberbullying: El Proyecto ConRed. Revista digital de la Asociación Convives, vol.3, 34-44. Recuperado el 22 de mayo de $2015 \quad$ desde http://www.educatolerancia.com/pdf/ACOSO\%20ENTRE\%20IGUALES_CIBERACOSO_ \%20Revista\%20Convives.pdf.

Quesada, J. y López, A. (2010). Estereotipos de género y usos de la lengua: un estudio en Educación Secundaria. Ensayos, Revista de la Facultad de Educación de Albacete, 25. Recuperado el 20 de junio de 2015 desde http://www.uclm.es/ab/educacion/ensayos.

Rodríguez-Gómez, J.M. (2008). Los docentes ante las situaciones de violencia escolar. Revista Electrónica Interuniversitaria de Formación del Profesorado, vol. 11 (3), 32-39. Recuperado el 20 de junio de 2015 desde http://www.aufop.com/aufop/home/

Rodríguez, Y., Lameiras, M., Carrera, M.V. y Vallejo, P. (2013). La fiabilidad y validez de la escala de mitos hacia el amor: las creencias de los y las adolescentes. Revista de 
Psicología Social: International Journal of Social Psychology, vol.28 (2), 157-168. Doi: 10.1174/021347413806196708.

Rubio, F., López, M.A., Saúl, L.A. y Sánchez-Elvira, A. (2012). Direccionalidad y expresión de la violencia en las relaciones de noviazgo de los jóvenes. Acción Psicológica, vol.9 (1), 61-70. Doi: http://dx.doi.org/10.5944/ap.9.1.437.

Ruiz-Pinto, E. García, R. y Rebollo M.A. (2013). Relaciones de género de adolescentes en contextos educativos. Análisis de redes sociales con perspectiva de género. Profesorado. Revista de curriculum y formación del profesorado, vol.17 (1), 123-140. Recuperado el 15 de mayo de 2015 desde http://www.ugr.es/local/recfpro/rev171ART8.pdf.

Serrano, M. y Pérez, M.D. (2011). Formación del profesorado de educación secundaria obligatoria sobre el bullying. Revista Española de Orientación y Psicopedagogía, vol.22 (1), 58-68.

Valverde, M.A. (2013). Actuaciones para sensibilizar y prevenir el acoso escolar en los centros. Revista digital de la Asociación Convives, vol.3, 64-73. Recuperado el 22 de mayo de 2015 desde http://www.educatolerancia.com/pdf/ACOSO\%20ENTRE\%20IGUALES_CIBERACOSO \%20Revista\%20Convives.pdf

Viejo, C. (2012). Dating Violence y Cortejo Adolescente. Un Estudio sobre la Violencia en las Parejas Sentimentales de los Jóvenes Andaluces. Córdoba: Universidad de Córdoba. 